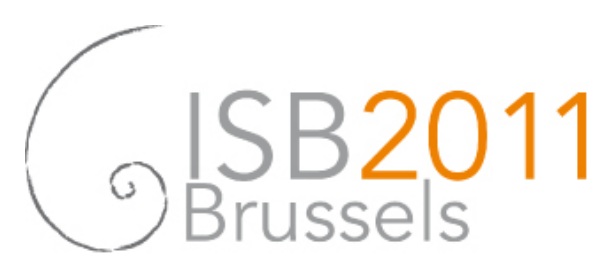

\title{
OPTIMIZING WHOLE-BODY KINEMATICS TO MINIMIZE VALGUS KNEE LOADING DURING SINGLE-LEG LANDING: IMPLICATIONS FOR ACL INJURY RISK
}

\author{
${ }^{1}$ Cyril J. Donnelly, ${ }^{1,2}$ David G. Lloyd, ${ }^{1}$ Bruce C. Elliott and ${ }^{3}$ Jeffrey A. Reinbolt, \\ ${ }^{1}$ School of Sport Science, Exercise and Health, The University of Western Australia, Australia; \\ ${ }^{2}$ Musculoskeletal Research Program, Griffith Health Institute, Griffith University, Australia: \\ ${ }^{3}$ Department of Mechanical, Aerospace and Biomedical Engineering, University of Tennessee, USA; \\ email: reinbolt@utk.edu,web: rrg.utk.edu
}

\section{SUMMARY}

The kinematics that cause elevated valgus knee loading and subsequent anterior cruciate (ACL) injury risk during singleleg landing (SLL) sport manoeuvres are not well understood. A subject-specific 37 degree-or-freedom (DoF) full-body torque-driven simulation of an athletic male during a SLL sport manoeuvre was created in OpenSim 1.9.1. A Reduced Residual Algorithm (RRA) was first used to create a dynamically consistent torque driven simulation of the weight acceptance phase of stance (WA) during SLL. RRA was used a second time to optimize the kinematics of SLL that reduces peak valgus knee torques, while maintaining dynamic consistency with experimentally recorded ground reaction forces and moments. Following kinematic optimization, mean peak externally applied valgus and internal rotation knee moments were reduced by $26 \%(23.3 \mathrm{Nm})$ and $10 \%(3.2 \mathrm{Nm})$ respectively. The simulation elevated contralateral leg hip $\left(7.8^{\circ}\right)$ and knee joint $\left(19.3^{\circ}\right)$ extension to decrease peak valgus knee moments during WA of SLL. The orientation of the whole-body centre of mass relative to the stance foot did not change. A larger sample size is currently being analyzed to determine if these findings are simulation specific or associated with a more generalized kinematic strategy to decrease peak valgus knee landing during SLL.

\section{INTRODUCTION}

Approximately one half of non-contact anterior cruciate ligament (ACL) injuries occur during landing sport

manoeuvres $[1,5]$, with the majority of these injuries occurring during single-leg landing (SLL) situations $[1,10]$. Anterior tibial translation, due to quadriceps driven extension of the knee combined with eternally applied valgus or internal rotation knee moments have been shown to elevate ACL strain in-vivo [6]. Motion analysis of SLL show that externally applied valgus knee moments during the weight acceptance phase of stance (WA) are elevated [9].

Interventions that aim to prevent ACL injuries should focused on reducing valgus knee moments during WA of SLL when injury risk is thought to be highest. For counter measures to be developed, causal relationships between whole-body (WB) kinematics and valgus knee loading must first be identified.

Using motion data of SLL and the Reduced Residual Algorithm (RRA) in OpenSim (SimTK.org, Stanford, CA), causal relationships between whole-body (WB) kinematics and peak valgus knee moments during WA of SLL can be identified [2].

\section{METHODS}

Three-dimensional full-body kinematics and ground reaction forces and moments (GRF) of an athletic male (height $1.74 \mathrm{~m}$; mass $70.2 \mathrm{~kg}$ ) conducting a SLL sport task were recorded in a laboratory setting. A scaled full-body 37 degree of freedom (DoF) skeletal model $[2,4]$ was then created in OpenSim.

The RRA within OpenSim 1.9.1 was used to produce a dynamically consistent torque driven simulation during the WA of SLL (peak residual forces $<250 \mathrm{~N}$ and moments $<150$ $\mathrm{Nm}$; RMS kinematic error $\leq 10^{\circ}$ ) [11]. Peak valgus knee torque was reduced and RRA was run again, producing a dynamic consistent optimized kinematic solution with reduced peak valgus knee torque [2].

Knee torques were expressed as externally applied knee moments. WB kinematics, knee moments and WB CoM position relative to the stance foot CoM were compared preto-post kinematic optimization.

The mean angular difference (deg) for all 37 DoF pre and post kinematic optimization were calculated in $20 \%$ intervals over WA. The mean angular difference of all joints across all time points during WA was then calculated. Any joint with a kinematic angular change (deg) greater than $2 \sigma$ above this mean was defined as a critical joint coordinate and identified as a kinematic change that most influenced the observed changes in peak valgus knee moments.

\section{RESULTS AND DISCUSSION}

Following WB kinematic optimization, peak valgus and internal rotation knee moments were reduced by $26 \%(23.3$ $\mathrm{Nm})$ and $10 \%(3.2 \mathrm{Nm})$ respectively (Figure 1). Peak flexion moments were elevated by 56\% (121.6 Nm). Increases in flexion knee moments combined with decreases in peak frontal plane knee moments have been observed following gait re-training in clinical settings $[3,12]$. Elevated flexion moments have been identified as an unlikely mechanism of ACL injury [7,8]. Results from this and previous literature suggest that in-silico changes in knee moments following 
kinematic optimization are consistent with clinical findings and effective in reducing ACL injury risk.

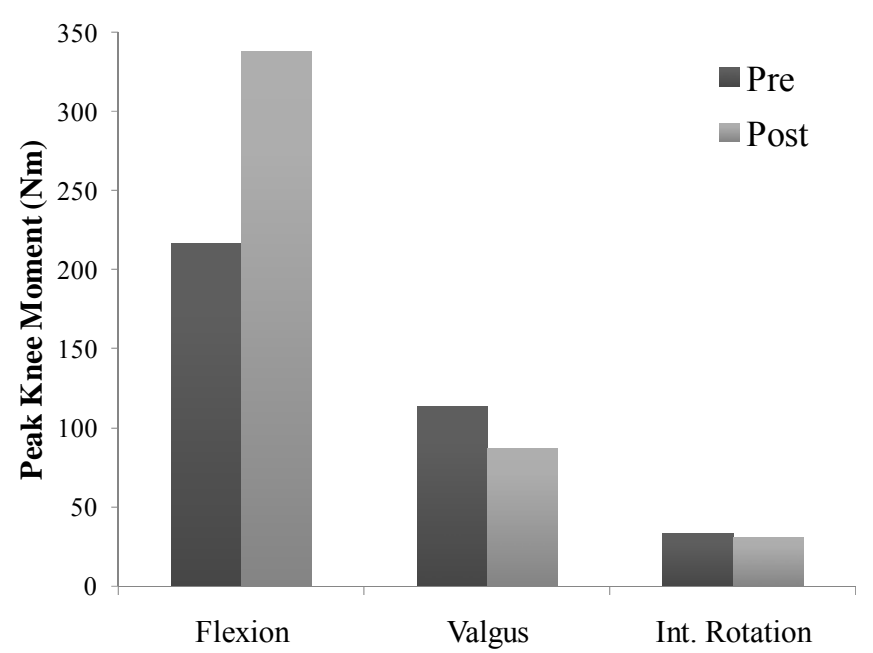

Figure 1: Peak flexion, valgus and internal rotation knee moments pre-to-post kinematic optimization calculated during the WA phase of SLL.

Contralateral leg hip $\left(7.8^{\circ}\right)$ and knee $\left(19.3^{\circ}\right)$ extension were the two primary kinematic differences associated with changes used to reduce peak valgus knee moments during WA of SLL (Figure $2 \& 3$ ).

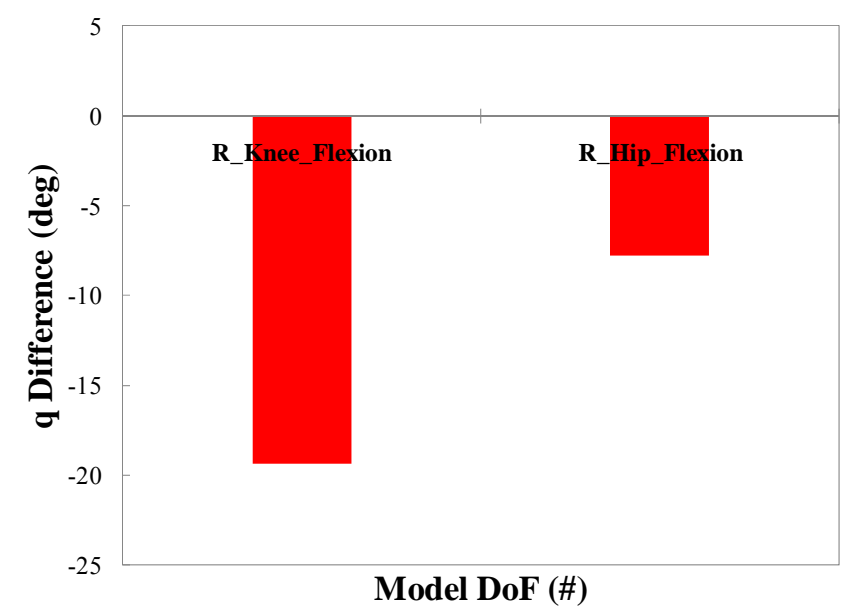

Figure 2: Difference in critical joint coordinates (deg) pre-topost kinematics optimization during the WA phase of SLL. Note: Flexion, abduction and internal rotation are positive.

The position of the WB CoM relative to stance foot CoM did not change. Elevated contralateral leg hip and knee joint extension likely produced a counter moment that influenced the changes in knee moments observed in the stance limb knee joint.

Due to the complex dynamic nature of SLL we are analyzing a larger sample to determine if these findings are simulation specific or associated with a general and/or consistent kinematic strategy.

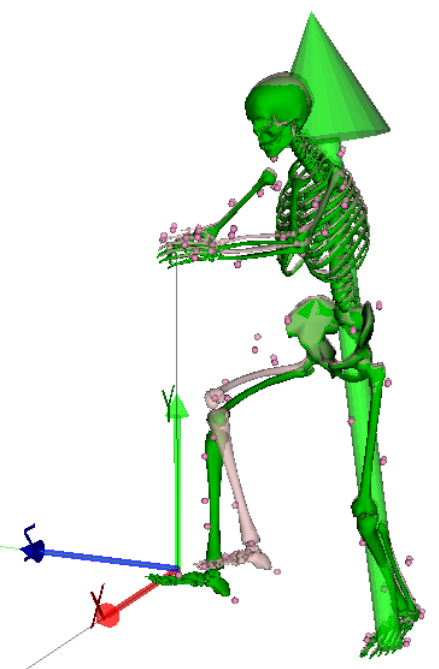

Figure 3: Depiction of SLL landing tasks before (red) and after (green) kinematic optimization used to minimize peak valgus knee moments. Note the relative increase in contralateral leg hip and knee extension following the kinematic optimization.

\section{CONCLUSIONS}

Contralateral leg hip and knee extension during the WA phase of SLL was used to decrease peak valgus knee loading and subsequent ACL injury risk during WA of SLL. Results also show that the Reduced Residual Algorithm within OpenSim can be used to identify causal relationships between an individual's kinematics and peak valgus knee loading during the WA phase of SLL.

\section{ACKNOWLEDGEMENTS}

We thank PAFIX through the NHMRC (grant number 400937) and U. Tennessee for funding this study. We also thank the CSB and the UWA Convocation office for CJD's travel funding and making this research collaboration possible.

\section{REFERENCES}

1. Cochrane JL, et al., J Sci Med in Sport. 10:96-104, 2007.

2. Donnelly CJ, et al., Proceedings of the $6^{\text {th }}$ WCB, Singapore, 2010.

3. Fregly BJ, et al., IEEE Trans Biomed Eng. 54(9):16871695, 2007.

4. Hamner SR, et al., J Biomec. 43(14):2709-16, 2010.

5. Krossaug T, et al., Am J Sport Med. 35:359-36, 2007.

6. Markolf KL, et al., J Orthop Res. 13(6):930-935, 1995.

7. McLean SG et al., Clin Biomech (Bristol, Avon). 19(8), 828-838, 2004.

8. McLean SG, et al., Clin Biomech. 23:926-93, 2008.

9. McLean SG, et al., Clin Biomech. 25:563-569, 2010.

10. Meuffels DE \& Verhaar JAN, Acra Orthopaedica. 79(4):515-518, 2008.

11. Reinbolt JA \& Donnelly CJ. Proceedings of the Eleventh International Symposium on the 3D analysis of Human Movement. San Francisco, California, 2010.

12. Walter, JP, et al., J Orthop Res. 28(10):1348-1354, 2010. 